\title{
Medical Image of the Week: Cheyne-Stokes Respiration on Overnight Polysomnography
}

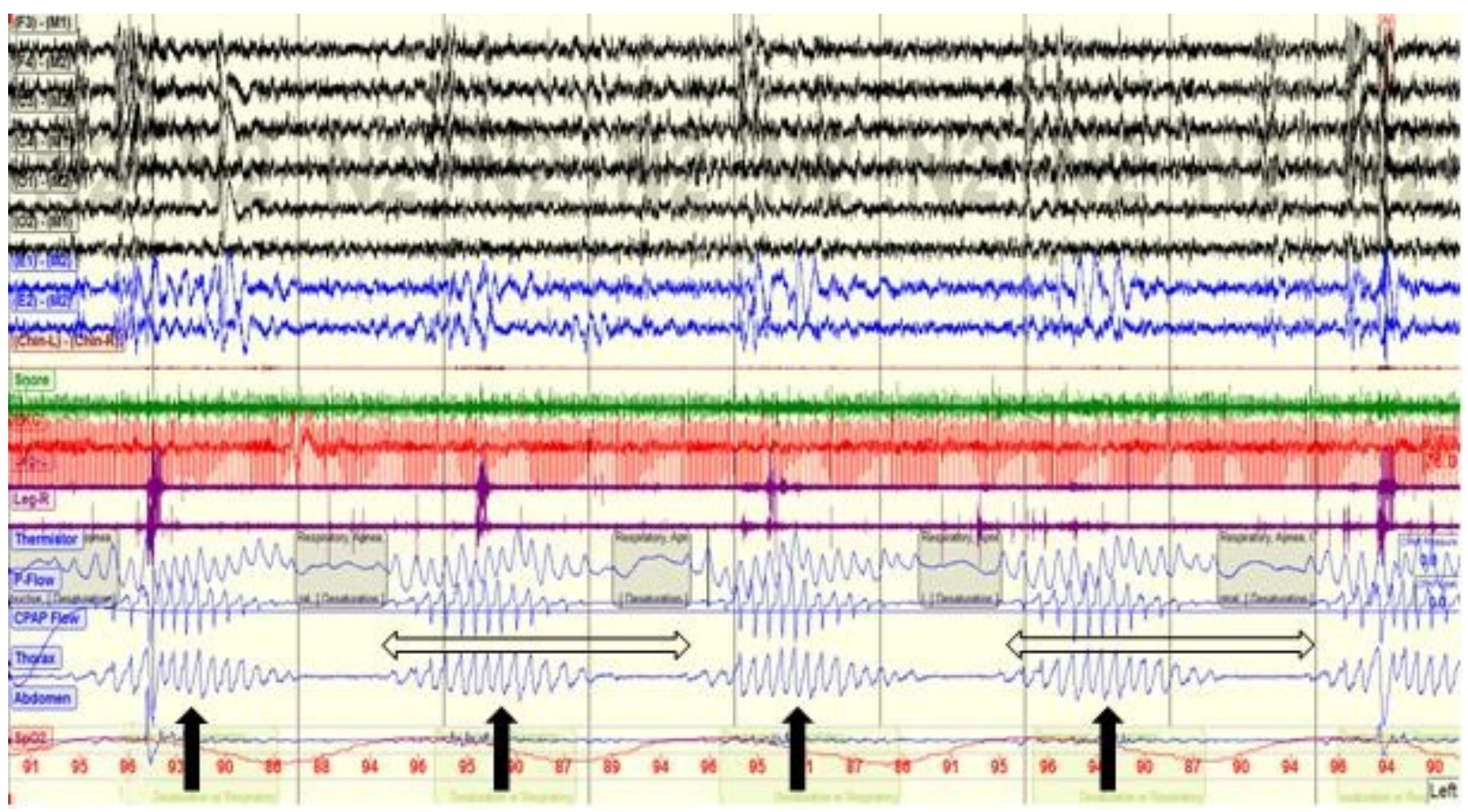

Figure 1. 300 second polysomnogram window showing crescendo-decrescendo pattern of Cheyne-Stokes respiration (solid black arrows). Cycle length is approximately 60 seconds in duration (Outlined black arrows).

A 75 year old man with a significant past medical history of atrial fibrillation, hypertension, complete heart block status-post pacemaker implantation, thoracic aortic aneurysm, and ischemic cardiomyopathy, was referred to the sleep laboratory for evaluation for suspected sleep disordered breathing. The patient had subjective complaints of morning headaches, reported apnea, un-refreshing sleep, nocturnal urination, and intermittent snoring. The diagnostic polysomnogram was significant for periodic breathing, Cheyne-Stokes pattern, with a cycle length that ranged from 60-65 seconds (Figure 1). Oxygen saturation nadir was $79 \%$ as measured by pulse oximetry. Electrocardiogram showed a persistently paced rhythm.

Cheyne-Stokes respiration is a periodic breathing pattern characterized by crescendodecrescendo episodes of respiratory effort that are interspersed between periods of apnea. It is typically seen in individuals with systolic heart failure, but can also be seen in those with intracerebral hemorrhage or infarction. The mechanism for Cheyne-Stokes respiration involves increased central controller gain causing increased central nervous system sensitivity to changes in arterial blood gas $\mathrm{PCO}_{2}$ and $\mathrm{PO}_{2}$. Increased circulation time results in circulatory delay between gas exchange occurring at the alveolar capillary membrane and the central chemoreceptors in the medulla. The result is instability in respiration (1). 
Ryan Nahapetian, MD, MPH and Sairam Parthasarathy, MD

Pulmonary, Allergy, Critical Care, \& Sleep Medicine

University of Arizona, Tucson, AZ

\section{Reference}

1. Quaranta AJ, D'Alonzo GE, Krachman SL. Cheyne-Stokes respiration during sleep in congestive heart failure. Chest. 1997;111(2):467-73. [CrossRef] [PubMed] 